\title{
Who Pays for Renewables? Increasing Renewable Subsidisation due to Increased Datacentre Demand in Ireland
}

\author{
Muireann Lyncha ${ }^{a}$ Mel T. Devine \\ Received: 12.03.2019; Revised: 12.04.2019; Accepted: 13.04.2019
}

\begin{abstract}
Demand from datacentres makes up a rapidly growing portion of electricity demand in Ireland. Increased demand, in turn, gives rise to increased renewable generation, mandated by government targets, and a corresponding increase in subsidisation levels. The current method of apportioning renewable subsidy costs may lead to consumers other than datacentres bearing this excess cost of subsidisation. This study calculates the expected impact on these consumers.
\end{abstract}

JEL codes: Q40, Q42, Q48, Q58

Keywords: Renewables; electricity; subsidy; datacentres

\section{Introduction}

Environmental concerns, particularly regarding carbon emissions, have driven many recent European energy policies. Chief amongst them is the policy of requiring that $20 \%$ of total final energy demand in European Union member States be met by renewable energy (EC, 2009). As a consequence, Ireland has set a target for $40 \%$ of electricity demand to be met by renewable generation, primarily wind energy (DCENR, 2009). In order to incentivise the deployment of sufficient renewable generation capacity to meet this target, renewable generators in Ireland are compensated by means of a subsidy payment. The subsidy currently takes the form of a feed-in tariff; specifically, when the market price drops below the tariff, the generator receives the tariff for each unit of electricity generated, while when the market price rises above the tariff, the generator receives the market price. The design of the feed-in tariff in Ireland can thus be considered a floor price. This mechanism shall soon be replaced by a new auction-based subsidy (DCCAE, 2017).

The revenues necessary to fund both the current feed-in tariff subsidy mechanism and the future auction-based subsidy mechanism are levied on consumers through a Public Service Obligation levy. The proportion of the levy paid by each consumer category is determined by the category's contribution to peak demand (eISB, 2002). The consumer categories in question are residential consumers, small commercial consumers, and medium and large

a Economic and Social Research Institute, Dublin-Ireland (e-mail: muireann.lynch@esri.ie).

b Economic and Social Research Institute and School of Electrical and Electronic Engineering, University College Dublin, Dublin-Ireland (e-mail: mel.devine@ucd.ie). 
profile consumers. The distributional implications of levying the Obligation on the basis of each group's peak demand is of interest in this research.

There has been a significant increase in the number of datacentres located in Ireland in recent years, with a corresponding increase in electricity demand. The total electricity demand from these datacentres is set to rise further still (EirGrid, 2017). Given that the target for renewable electricity is set as a proportion of the total electricity demand, this increase in electricity demand from datacentres necessitates higher absolute levels of renewable generation in order to meet the targets, with a corresponding rise in renewable subsidisation. However, apportioning the subsidisation cost on the basis of peak demand means consumers that did not give rise to the increase in renewable subsidisation must nevertheless bear the cost of same. This could give rise to equity concerns and potentially feed public opposition to renewable energy projects and/or new datacentres. Thus, a quantification of the total expected increased subsidy cost arising from the increased electricity demand from datacentres, and the division of this increase amongst the various consumer categories, is of interest to policymakers and electricity consumers. On the basis of our results, we propose that an alternative method for distributing the cost of subsidisation be explored, which would see the cost incurred by datacentres being borne by consumers in that sector only.

\section{Calculation of Impact of Datacentres}

The present value of the PSO cost over the lifetime of the renewable capacity investment is calculated in the same manner as in Devine et al. (2017). The cost is calculated for three different levels of renewable capacity, corresponding to three different levels of demand, which are largely driven by the rollout of datacentres. These different levels of datacentre connection are estimated by the Transmission System Operator of Ireland (EirGrid, 2017).

While the future Irish renewable generation portfolio is unknown, renewable energy policy has moved in the direction of specifically subsidising diverse sources of renewable generation rather than focusing on the least-cost technology (DCCAE, 2017). In particular, the Government's Energy White Paper states that "The deployment of solar in Ireland has the potential to increase energy security, contribute to our renewable energy targets, and support economic growth and jobs" (DCENR, 2015). With this in mind, we calculate the expected increased subsidy cost twice, assuming in the first instance that the extra renewable generation is met by onshore wind generation, and in the second instance that it is met by photovoltaic solar generation. We apportion this expected cost increase across the different consumer categories according to the current method of PSO cost allocation, which is expected to continue under the new auction-based mechanism.

The majority of renewable generation in Ireland at present is met by onshore wind (EirGrid, 2017). We assume the onshore wind required to meet the underlying renewable target in the low demand scenario, which corresponds to a low connection rate of datacentres, is 3942MW (calculated from EirGrid (2017)). This level of onshore wind generation is assumed to exist also in the median and high demand scenarios and so we calculated the cost of the extra renewable generation, arising primarily from datacentres, required under the median and high demand scenarios twice. In the 'wind' scenario we assume the extra renewable generation is met by extra onshore wind generation and in the 'solar' scenario we assume the extra renewable generation is met by photovoltaic solar generation. The extra renewable capacity required under the median and high demand scenarios is calculated 
using a capacity factor of $31 \%$ for onshore wind, as per EirGrid (2017), and $9 \%$ for solar (Ryan et al., 2016). The demand and renewable generation capacities required (on top of the 3942MW of onshore wind in the low demand scenario) are given in Table 1. Note that these figures apply to the Republic of Ireland only rather than the whole all-island market of Ireland.

We do not make any assumptions regarding the ownership of the renewable generation capacity, and we assume that all renewable generation capacity benefits from the subsidy scheme. Thus while datacentre owners may invest in renewable generation themselves, we assume investments will qualify for and receive, a subsidy, and that datacentre owners will contribute to the cost of the total subsidy according to the current arrangements. In reality, datacentre owners may choose to waive their entitlement to a subsidy, but their requirement to contribute to the subsidisation of other renewable generation will remain unchanged. Thus the total level of subsidisation may be affected by a datacentre owner declining their share of the subsidisation revenues but the proportional effects, which are of interest here, remain unchanged.

Table 1: Demand (TWh) and renewable generation capacities (MW) scenarios

\begin{tabular}{lrrr}
\hline & Low demand & Median demand & High demand \\
\hline Demand (TWh) & 30 & 31.2 & 32.6 \\
Wind scenario (MW) & - & 157.7 & 341.7 \\
Solar scenario (MW) & - & 543.2 & 1176.9 \\
\hline Source:
\end{tabular}

Source: Authors' calculations.

The total expected cost of subsidising the different levels of renewable capacity over twenty years is calculated using, for the most part, the same cost and technical assumptions as Devine et al. (2017). In addition, the feed-in tariff for wind is set at $€ 72 / \mathrm{MWh}$, as this was the level of the tariff in 2017. At present solar generation does not have a feed-in tariff, so we used the figure of $€ 130 /$ MWh from Ryan et al. (2016).

The total expected cost under each scenario is shown in Table 2.

Table 2: Total subsidy cost for each of renewable installation scenario $(\mathrm{M} €)$

\begin{tabular}{lrrr}
\hline PSO costs & Low demand & Median demand & High demand \\
\hline Wind scenario & 3092 & 3171 & 3307 \\
Solar scenario & 3092 & 3708 & 4352 \\
\hline
\end{tabular}

Source: Authors' calculations.

As expected, higher renewable generation levels give rise to higher costs, with the solar scenario giving rise to higher costs again. Table 3 shows the contribution to peak demand by each consumer category in 2016, according to CER (2016). We calculate the new contribution from each under the median and high demand scenarios, taking the new demand from datacentres into account, and assuming there is no change in demand from other consumers.

Given these shares, the proportion of the subsidy cost in Table 2 can be divided between the various consumer categories. In particular, the extra subsidy cost attributable to the demand from datacentres is decomposed between the three consumer categories in Table 4.

Focusing on residential consumers, the increase in annual PSO costs they bear is $3 \%$ and $7 \%$ for the median and high demand scenarios, relative to the low demand scenario, when the increased renewable generation is provided by onshore wind generation. If the extra 
Table 3: Share of peak demand made up by each consumer category under each demand scenario (\%)

\begin{tabular}{lcrc}
\hline Consumer category & Low & Median & High \\
\hline Small Commercial & 10.6 & 10.31 & 10.08 \\
Residential & 36.8 & 35.79 & 34.99 \\
Medium and High Profile & 52.6 & 53.9 & 54.93 \\
\hline Source: Authors' calculations. & & &
\end{tabular}

Table 4: Portion of extra costs borne by each consumer group (reference scenario: low demand wind scenario) $(\mathrm{M} €$ )

\begin{tabular}{lrrrr}
\hline PSO costs & \multicolumn{2}{c}{ Median } & \multicolumn{2}{c}{ High } \\
\hline & Wind & Solar & Wind & Solar \\
Small Commercial & 8.3 & 65.22 & 22.7 & 133.5 \\
Residential & 28.8 & 226.4 & 78.9 & 463.4 \\
Medium and High Profile & 41.2 & 323.7 & 112.7 & 662.3 \\
\hline Source: Authors' calculations. & & & &
\end{tabular}

generation is provided by solar, the annual increase in the PSO costs is much higher, at $20 \%$ for the median demand scenario and $41 \%$ for the high demand scenario. Given that our analysis assumes the residential consumer does not change their demand at all, any increase in PSO costs for these consumers, however small, could be seen as an unfair imposition on these consumers. This inequity is even more pronounced considering that datacentres based in Ireland will service their consumers all over the EMEA area and yet Irish consumers would bear the cost of the associated renewables.

This change in PSO cost burden has its roots in the PSO mechanism design. The required quantity of renewables is determined on the basis of total demand rather than total capacity. Apportioning the cost on the basis of peak demand thus places a greater share of the burden on consumers with a more variable demand pattern. As industrial loads in general, and datacentres in particular, have relatively fixed demand profiles, they consequently bear only a portion of the subsidisation cost for which their demand is responsible. Apportioning PSO cost according to the contribution of each sector to average rather than peak demand instead would mean each sector would be responsible for the proportion of PSO costs that arose due to their contribution to market demand.

\section{Conclusion}

This brief article calculated the increased renewable subsidy costs that increased loads from datacentres may impose on consumers in Ireland. The extra cost arises from EU mandated government targets for renewable generation, which are calculated as a portion of total demand. Assuming the current method of apportioning PSO costs is applied as these new loads come on stream, residential and small commercial consumers will face increased PSO payments. The level of these costs increases considerably if the extra renewable requirement is met with solar generation.

Datacentre owners may prove unwilling to inflict higher costs on consumers, through PSO payments or otherwise. Therefore datacentre owners and/or energy regulators may wish to explore an alternative method of calculating and apportioning PSO costs to new loads of this type. The findings suggest that such an approach is prudent and may inform discussions on an alternative mechanism. 


\section{References}

CER. (2016). Public Service Obligation Levy 2016/17 (Policy Document). Commission for Energy Regulation (CER). https://www.cru.ie/wp-content/uploads/2016/ 07/CER16152-PSO-Levy-2016-17-Proposed-Decision-Paper .pdf.

DCCAE. (2017). Renewable Electricity Support Scheme (RESS) High Level Design (Policy Document). Department of Communications, Climate Action and Environment (DCCAE). https://www.dccae.gov.ie/documents/RESS\%20Design\%20Paper.pdf.

DCENR. (2009). National Renewable Energy Action Plan Ireland (Policy Document). Department of Communications, Energy and Natural Resources (DCENR). https://www.dccae.gov.ie/documents/The\%20National\% 20Renewable\%20Energy\%20Action\%20Plan\%20 (PDF). pdf.

DCENR. (2015). Ireland's Transition to a Low Carbon Energy Future 20152030 (Report). Department of Energy, Communications and Natural Resources (DCENR). https://www.dccae.gov.ie/en-ie/energy/publications/Documents/ 2/Energy \%20White\%20Paper\%20-\%20Dec\%202015.pdf.

Devine, M. T., Farrell, N., \& Lee, W. T. (2017). Optimising Feed-in Tariff Design Through Efficient Risk Allocation. Sustainable Energy, Grids and Networks, 9, 59-74. doi:10.1016/j.segan.2016.12.003

EC. (2009). The promotion of the use of energy from renewable sources and amending and subsequently repealing directives 2001/77/ec and 2003/30/ec. [Directive 2009/28/EC of the European Parliament and of the Council of 23 April 2009]. http://eur-lex. europa.eu/LexUriServ/LexUriServ. do?uri=0J:L : 2009: 140:0016:0062: EN : PDF.

EirGrid. (2017). All-Island Generation Capacity Statement 2017-2026 (Policy Document). EirGrid plc. http://www.eirgridgroup.com/site-files/library/EirGrid/\%4289 EirGrid_GenCapStatement_v9_web.pdf.

eISB. (2002). S.I. No. 217/2002 - Electricity Regulation Act 1999 (Public Service Obligations) Order 2002 (Policy Document). electronic Irish Statute Book (eISB). http://www.irishstatutebook.ie/eli/2002/si/217/made/en/print.

Ryan, L., Dillon, J., La Monaca, S., Byrne, J., \& O'Malley, M. (2016). Assessing the System and Investor Value of Utility-scale Solar PV. Renewable and Sustainable Energy Reviews, 64, 506-517. doi:10.1016/j.rser.2016.06.004 